\title{
LISA - AN ESA CORNERSTONE MISSION FOR THE DETECTION AND OBSERVATION OF GRAVITATIONAL WAVES
}

\author{
K. Danzmann ${ }^{1,2}$ and the LISA Study Team \\ ${ }^{1}$ Institut für Atom- und Molekülphysik, Universität Hannover, Appelstr. 2, 30167 Hannover, Germany \\ ${ }^{2}$ Max-Planck-Institut für Quantenoptik, Hans-Kopfermann-Str. 1, 85748 Garching, Germany
}

\section{ABSTRACT}

The primary objective of the Laser Interferometer Space Antenna (LISA) is to detect and observe gravitational waves from massive black holes, galactive binary stars, and violent events in the Universe in a frequency range from $10^{-4}$ to $10^{-1} \mathrm{~Hz}$ which is totally inaccessible to ground based experiments. It uses highly stabilised laser light (Nd:YAG, $\lambda=1.064 \mu \mathrm{m})$ in a Michelson-type interferometer arrangement.

A cluster of six spacecraft with two at each vertex of an equilateral triangle is placed in an Earth-like orbit at a distance of $1 \mathrm{AU}$ from the Sun, and $20^{\circ}$ behind the Earth. Three subsets of four adjacent spacecraft each form an interferometer comprising a central station, consisting of two relatively adjacent spacecraft $(200 \mathrm{~km}$ apart), and two spacecraft placed at a distance of $5 \times 10^{6} \mathrm{~km}$ from the centre to form arms which make an angle of $60^{\circ}$ with each other. Each spacecraft is equipped with a laser.

A descoped LISA with only four spacecraft has undergone an ESA assessment study in the M3 cycle, and the full 6-spacecraft LISA mission has now been selected as a cornerstone mission in the ESA Horizons 2000 programme.

The LISA Assessment Report is available as ESA document SCI(94)6, May 1994. Detailed information on the LISA cornerstone mission is contained in the LISA Pre-Phase A Report, available as MPQ Report MPQ 208 (1996) from the Max-Planck-Institut für Quantenoptik. @ 2003 Published by Elsevier Ltd on behalf of COSPAR.

\section{OVERVIEW}

Conceptually, the idea of implementing an interferometer in space is straightforward, but the practical realisation requires an intricate blend of optical technology, spacecraft engineering and control. For a start, the interferometer mirrors can not simply float freely in space - they must be contained inside spacecraft. Nonetheless, they can be arranged to be floating almost freely inside the spacecraft, protected from external disturbances by the spacecraft walls. As long as the spacecraft do not disturb the mirrors, then, ideally, only gravitational waves would perturb their relative motion. "Drag-free control" can be employed to ensure that the spacecraft always remain centred on the mirrors.

In principle, then, the Michelson interferometer could be realised using three spacecraft: one at the "corner" to house the light source, beam splitter, and detector, plus one at each "end" to house the remote mirrors. But there would be immediate practical problems with such a configuration. All three spacecraft would drift around, and the corner spacecraft would not be able to keep itself aligned with both of the end spacecraft at the same time. One way around this would be to have steerable optics inside the corner spacecraft so that 
alignment could be maintained with the two arms independently. To avoid this complexity, LISA uses six spacecraft, arranged in a triangular configuration with two at each vertex. With this setup, each of the corner

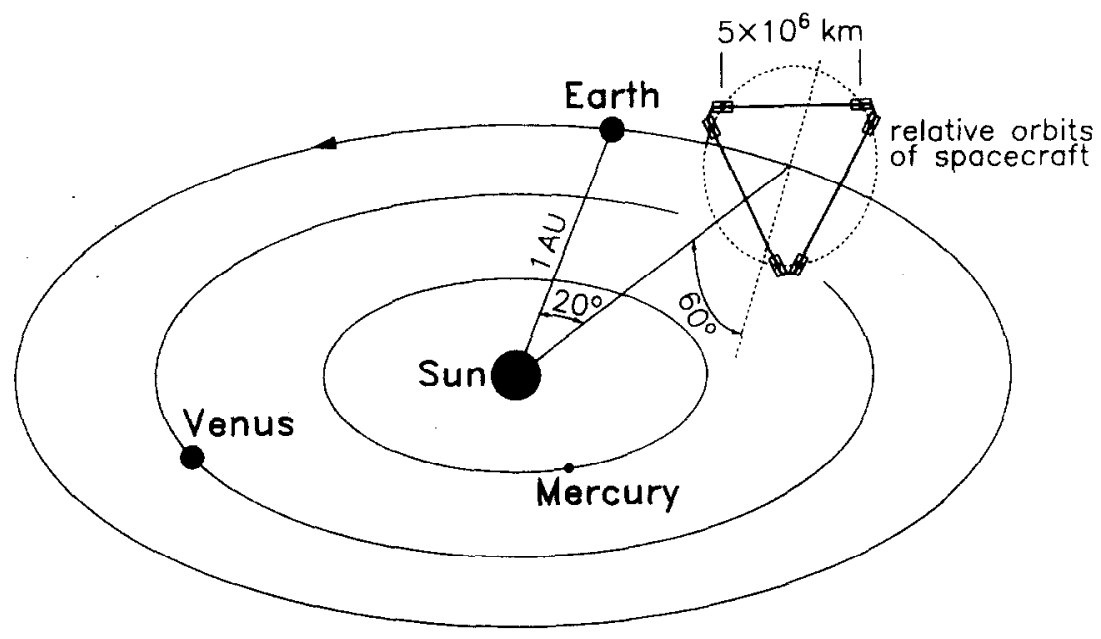

Figure 1 LISA concept. Six spacecraft in a triangle, with a pair at each vertex. Only four are required for the basic interferometry. The other two provide supplementary science information and some redundancy.

spacecraft can dedicate itself to pointing at only one of the end spacecraft, thus eliminating the need to steer the main optics. The corner spacecraft must, nevertheless, communicate with each other using steerable optics - but the separation distance is so much less that the steerable components can be much smaller, and hence more manoeuverable.

Each "corner" pair of spacecraft, separated by $200 \mathrm{~km}$, is located at the vertex of a large triangle whose sides measure $5 \times 10^{6} \mathrm{~km}$ in length. This arm length has been chosen to optimise the sensitivity of LISA at the frequencies of known and expected sources. A factor of 2 increase may be desirable. However, an arm length increase beyond that would begin to compromise the high- frequency sensitivity when the light in the arms experiences more than half of the gravitational wave period. An interferometer shorter than $5 \times 10^{6} \mathrm{~km}$ would begin to lose the interesting low-frequency massive blackhole sources. It would give less scientific information but would not be any easier to build or operate because the spacecraft and the interferometry would be essentially the same.

Nominally in such an arrangement of spacecraft, any two sides of the triangle (i.e. four spacecraft) can be used for the main interferometry, with the third arm giving supplementary information and redundancy. With the six spacecraft configuration, up to two can be lost without jeopardising the mission (as long as the two failures are not at the same corner), since the basic group of four in an approximate " $L$ " shape is sufficient to perform the full interferometry.

Each spacecraft is actually in its own orbit around the Sun. The six individual orbits have their inclinations and eccentricities arranged such that, relative to each other, the spacecraft rotate on a circle 'drawn through' the vertices of the giant triangle which is tilted at $60^{\circ}$ with respect to the ecliptic. With this special choice of orbits, the triangular geometry of the interferometer is largely maintained throughout the mission. The centre of the triangle is located on the ecliptic $-20^{\circ}$ behind the Earth - and follows the Earth on its orbit around the Sun. Ideally, the constellation should be as far from Earth as possible in order to minimise gravitational disturbances. The choice of $20^{\circ}$ is a practical compromise based on launch vehicle and telemetry capabilities. 


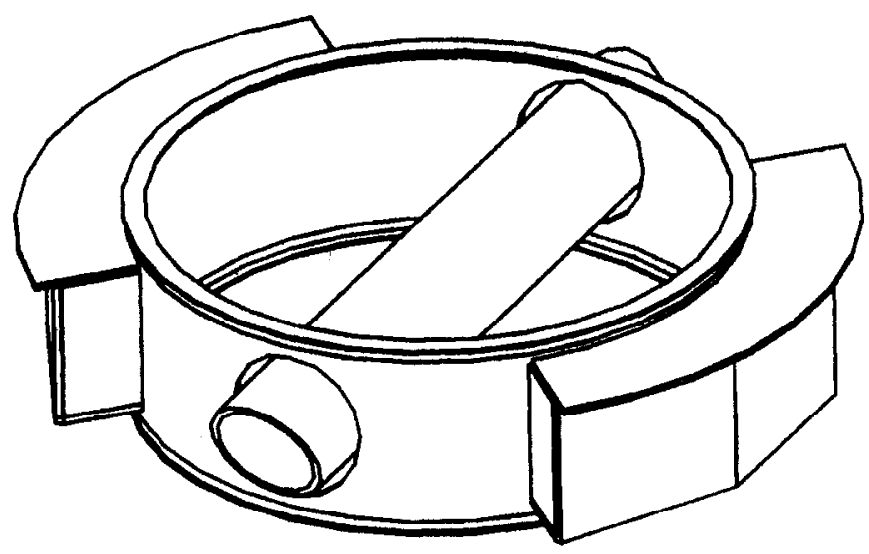

Figure 2 One of the six identical LISA spacecraft. The main structure is a ring with a diameter of $2.6 \mathrm{~m}$, and a height of $0.7 \mathrm{~m}$, made from carbon-epoxy for low thermal expansion. The ring supports the payload cylinder, as shown. Equipment boxes are mounted on the outside of the ring, to house non-precision electronics (e.g. power regulator, computer, radios). FEEP control thrusters (not shown) are mounted at various locations on the outer spacecraft structure. The tops of the equipment boxes support two annular sections of solar array for power generation. A lid on top of the spacecraft (not shown) protects the thermal shields and payload cylinder from direct sunlight.

The once-per-year orbital rotation of the LISA constellation around the Sun provides the instrument with angular resolution, i.e. the ability to pin-point the particular direction to a source. An interferometer is rather omnidirectional in its response to gravitational waves. In one sense this is advantageous - it means that more sources can be detected at any one time - but it has the disadvantage that the antenna cannot be "aimed" at a particular location in space. For a given source direction, the orbital motion of the interferometer Doppler-shifts the signal, and also affects the observed amplitude. By measuring these effects the angular position can thus be determined. This is analogous to the technique used by radio astronomers to determine pulsar locations.

It is expected that the strongest LISA sources (from very distant supermassive black holes) should be resolvable to better than an arcminute; and even the weaker sources (galactic binaries) should be positioned to within one degree throughout the entire galaxy.

A LISA spacecraft is shown in Fig. 2, and a cross-section of the payload in Fig. 3. Each spacecraft has its own I W laser (actually two, one for redundancy), its own two-mirror telescope for sending and receiving light,

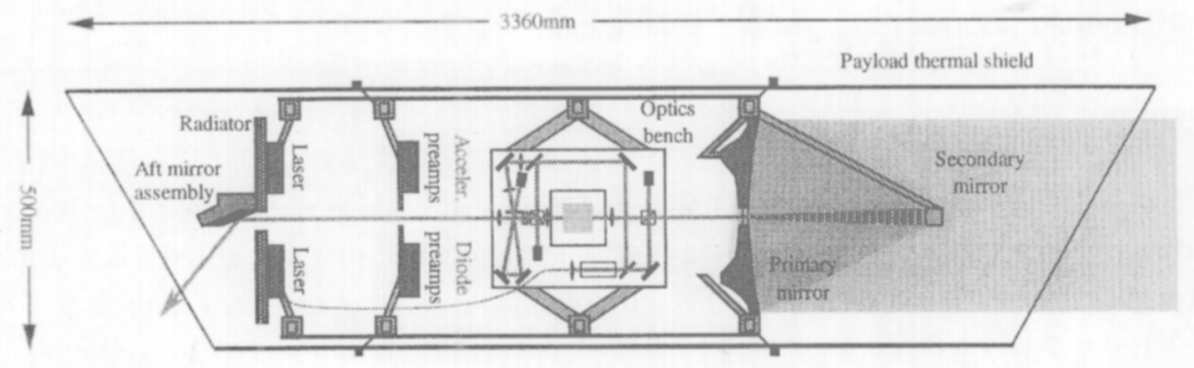

Figure 3 Cross-section of the payload on each of the six identical LISA spacecraft. 
and an optical bench which is a mechanically-stable structure on which various sensitive optical components are mounted. The mirrors enclosed in each spacecraft are actually $40 \mathrm{~mm}$ gold-platinum cubes (also referred to as the 'proof masses'). Each one is located inside a titanium vacuum can at the centre of the respective optical bench. Quartz windows allow access for the laser light.

Within the corner pair of spacecraft, one laser is the 'master', and a fraction of its light $(10 \mathrm{~mW})$ is bounced off the back surface of its cube, and sent to the neighbouring corner spacecraft (via the small steerable optics). where it is used as a reference to 'slave' the local laser. In this way, the main ( $\sim$ I W) beams going oul along each arm can be considered as having originated from a single laser. This is vital to the function of the interferometer.

The light sent out along an arm is received by the end spacecraft telescope, bounced off its cube, then amplified using its local laser, in such a way as to maintain the phase of the incoming light. The amplified light is then sent to the corner spacecraft. Amplification at the end spacecraft is required due to divergence of the beam over the very large distances. Even though each outgoing beam is extremely narrow - a few micro radians - it is about $20 \mathrm{~km}$ wide when it reaches the distant spacecraft. This diffraction effect, together with unavoidable optical losses, means that only a small fraction of the original output power $\left(\sim 10^{-10}\right)$ finally reaches the end diode. If this was simply reflected and sent all the way back, only about 200 photons per hour would reach the corner diode after the round-trip. The phase-signals they carry would be swamped by shot noise, the quantum-mechanical fluctuations in the arrival times of the photons. The amplification brings the number back up to over $10^{8}$ photons per second — which makes the signal detection straightforward using standard photodiodes. The phase precision requirement for this measurement is seven orders of magnitude less demanding than is routinely achieved (at higher frequencies) in ground-based prototype interferometers.

The resulting round-trip journey from the corner to the end and back, defines one arm of the large interferometer. On its return to the corner spacecraft, the incoming light is bounced off the cube and then mixed with a fraction of the outgoing light on a sensitive photodetector, where interference is detected. The resulting brightness variations contain the phase-shift information for one arm of the interferometer. This signal is then compared (in software on the on-board computer) with the corresponding signals from the other two arms, and some preliminary data processing is done. The results are then transmitted to Earth by radio link.

The LIS $\Lambda$ spacecraft must be designed to minimise the total mass and required power. Preliminary results yield a mass, per spacecraft, of $300 \mathrm{~kg}$, and an operational power requirement, per spacecraft, of $192 \mathrm{~W}$.

\section{THE PAYLOAD CYLINDER}

The telescope assembly, a preamplifier disk, a radiator disk, and the optical bench for the interferometry are mounted on a carbon-epoxy payload support cylinder with four stiffening rings surrounded by a carbonepoxy payload thermal shield cylinder. A shield, cut at a $30^{\circ}$ angle at both ends, keeps sunlight from the thermally stable payload interior throughout the heliocentric orbit.

\section{Telescope Assembly}

The receiving and transmitting telescope is a Cassegrain system with integral matching lens mounted from the payload support cylinder and protected by a thermal shield. The primary mirror is a double-arch lightweight ultra-low expansion ULE design and has a diameter of $38 \mathrm{~cm}$ and a focal length of $38 \mathrm{~cm}$. The secondary mirror, supported by a three-leg carbon-epoxy spider, is mounted $35 \mathrm{~cm}$ from the primary and has a diameter of $4 \mathrm{~cm}$ and focal length $3.3 \mathrm{~cm}$. The beam to the secondary mirror from the instrument package is expanded to a diameter of approximately $3 \mathrm{~cm}$ by a suitable lens in the plane of the primary mirror. The 
optical elements may have to be aspherics to reduce aberration in the $f / 1$ telescope and require carcful positioning. The temperature noise of the telescope must be less than $10^{-5} \mathrm{~K} / \sqrt{\mathrm{Hz}}$ at $10^{-3} \mathrm{~Hz}$ to achieve the desired performance. Active focus control may be required to compensate for any long-term temperiture drifts. The required final quality of the plane wavefront leaving the telescope is $\lambda / 15$.

\section{The Laser}

The light source is a ring laser consisting of two monolithic YAG (yttrium-aluminium-garnet) crystals in series, each pumped by two laser diodes. The nominal single-mode output power is $2 \mathrm{~W}$ at a wavelength ol $1061 \mathrm{~nm}$. For LISA this has been down-rated to $1 \mathrm{~W}$ to improve lifetime and ageing properties. The operalling temperature for the pump diodes and the YAG-crystal will be maintained by heaters. A complete spare laser will be carried on each spacecraft.

YAG lasers are both light weight and, for a laser, very efficient. The whole laser, including pump diodes, weighs less than a kilogram and will give a continuous optical output power of $1 \mathrm{~W}$ for an electrical input to the diodes of $18 \mathrm{~W}$. Furthermore, for a mission duration of a few years, the maintenance-free lifetime of the lasers has to exceed 100000 hours in the space environment. Diode-pumped Nd:YAGs have already demonstrated achievable lifetimes of 20000 hours at maximum power, and these lifetimes are considerably improved by operating the laser at half-power.

\section{The Radiator Disk}

The radiator disk (a carbon-plate $40 \mathrm{~cm}$ in diameter and $1 \mathrm{~cm}$ thick) is designed to radiate away the healt $(\approx 20 \mathrm{~W})$ dissipated by the laser. The aft-mirror assembly, for communication between the near spacecraft, is attached to this radiator disk.

\section{The Optical Bench}

The main optical components are located on an 'optical bench', containing the laser beam injection, detection and beam shaping optics, and the drag-free sensor (or "accelerometer"). 'The proof mass of the drag-free sensor acts as the mirror at the end of the interferometer arm. The bench consists of a solid ULE plate to which all components are rigidly attached. The components are shown schematically in Figure 4 . Most components on this structure are passive. Exceptions are a motorised positioner for fibre selection and focusing, photodiodes for signal detection and a phase modulator that allows transfer of information between craft. Light from the laser is delivered to the optical bench by a single-mode fibre. A second fibre coupled to the back-up laser is also provided and may be selected if required.

About $1 \mathrm{~mW}$ is split off the main light beam to serve as the local reference for the heterodyne measurement of the phase of the transponded beam returning from the far spacecraft. This splitting is performed by the finite transmission of the polarising beamsplitter in front of the main mirror. $\Lambda$ lso, in each craft, a few $\mathrm{mW}$ is split off and directed towards a triangular cavity. This cavity is used as a frequency reference in the master craft, with those in the other craft being available for backup purposes.

The incoming light from the telescope is reflected off the proof mass and superimposed with the local laser on the phase measuring diode. An optical isolating arrangement consisting of a polarising beamsplitter and a quarter-wave plate is used to allow the required transmission, reception and phase comparison functions to be carried out in a compact way. On the two spacecraft at a vertex a small fraction $(10 \mathrm{~mW})$ of the laser light is reflected off the back of the proof mass and sent to the other vertex spacecraft for phase-comparison with the near spacecraft via the steerable aft-mirror of $1 \mathrm{~cm}$ diameter. This mirror is servoed using the sig- 


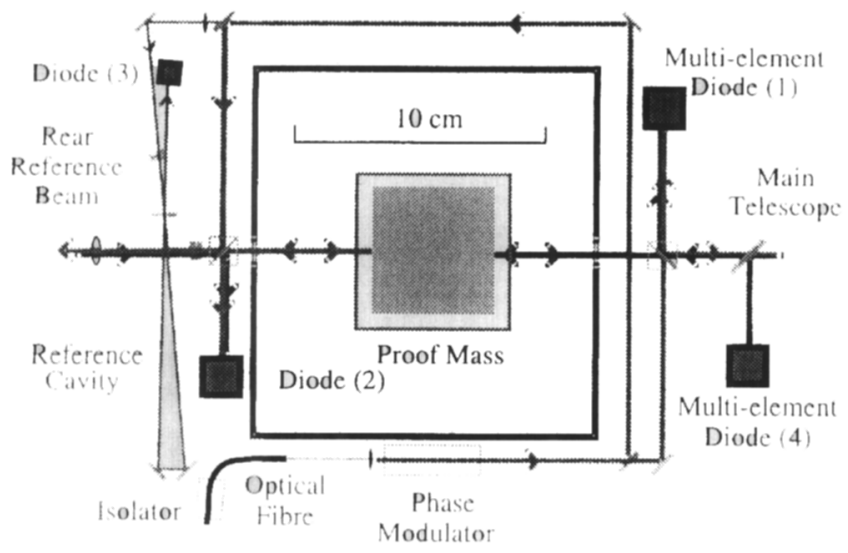

Figure 4 Diagram the optical bench. The chosen light path renders the measurement insensitive to movement on the spacecraft. The path followed by light from the local laser is shown in grey and that for light received from distant spacececraft is shown in black. Diode 1 registers the main interferometer signal and diode 2 the signal from the other spacecraft at that vertex, diode 3 is used to lock the laser to the reference cavity and diode 4 is used in pointing the spacecraft.

nal from an auxiliary quadrant photodiode which senses both the phase difference between the two beams and the direction of the incoming beam from the other central spacecraft. By bouncing the laser beams olf the proof mass in the manner described, the interferometric measurement of proof mass position is, to first order, unaffected by motion of the surrounding spacecraft. This allows a relaxation of its relative motion specification (though the requirement on proof mass residual motion with respect to inertial space remains unchanged).

\section{Optical Efficiency}

There are two classes of effect that limit the efficiency of the transmission of light from the emitting laser to the detection diode on the far spacecraft. These are the divergence of the laser beam and losses in the various optical components.

Beam Divergence. Even the best collimated laser beam will still have some finite divergence governed by the size of the final optic. With a Gaussian beam optimised for transmission between mirrors of diameter $D$, with an arm length $L$, and a transmitted power $P$, the power received at the far craft is given by

$$
0.50 \frac{D^{4}}{\lambda^{2} L^{2}} P
$$

This is the case when the Gaussian beam has a waist (of radius $w$ ) at the transmitting craft that almost fills the final telescope mirror, $w=0.446 D$.

Efficiency of the Optical Chain. There are a large number of components in the optical chain. The main ones contributing to a loss of transmitted power are the fibre, the quantum efficiency, splitters, mirrors and lenses, and interference. All other components in the optical chain are assumed to be perfect. This gives a total transmission of the optical chain of .30. The term for interference is to allow for the fact that some signal is lost due to the imperfect matching of the local reference beam and the received light from the far 
craft: the local reference beam is Gaussian and the received beam is a 'Top Hat' mode.

The Preamplifier Disk

The preamplifier disk is a carbon-carbon structure on which the accelerometer preamplifiers, the photo-diode preamplifiers and an ultrastable oscillator USO are mounted. All other payload electronics are localed outside the payload cylinder, on the spacecraft structure.

Thermal Stability

A high level of thermal stability is required by the interferometer. Thermal variation of the optical cavity to which the lasers are stabilized introduces phase variations in the interferometer signal, which have to be corrected for by using data from the two arms separately. Thermally induced variations in the dimensions of the transmit/receive telescope will lead to changes in the optical path length. Variations in the dimensions of the spacecraft will change the positions of components which cause a change in the mass distribution and hence cause an acceleration of the test mass.

The thermal stability needed is achieved by using structural materials with low thermal expansion coefficient and by using multiple stages of thermal isolation. The spacecraft and payload structural elements will be made of composite materials with thermal expansion coefficient less than $1 \times 10^{-6} / \mathrm{K}$. The optical bench and telescope are supported by the payload cylinder which is weakly thermally coupled to the payload thermal shield which in turn is weakly coupled to the spacecraft body. This provides three stages of thermal isolation for the payload from solar and spacecraft electronics thermal input.

The main source of thermal variation is die to changes in the solar intensity its mean value of $1350 \mathrm{~W} \mathrm{~m}$. Observed insolation variations from $0.1 \mathrm{mHz}$ to $10 \mathrm{mHz}$ can be described by a spectral density with a shallow frequency dependence:

$$
1.75 \times\left(\frac{\mathrm{f}}{1 \mathrm{mHz}}\right)^{1 / 3} \mathrm{~W} \mathrm{~m}^{-2} / \sqrt{\mathrm{Hz}} .
$$

To quantify the effects of solar and electrical variations, a simple thermal model for the spacecraft was formed with single nodes for the spacecraft body, solar panels, optical bench, telescope, laser radiator and electronics disk. The temperature fluctuations of the optical bench due to solar fluctuations were found to be well under the value of $10^{-6} \mathrm{~K} / \sqrt{\mathrm{Hz}}$ at $1 \mathrm{mHz}$ used in the analysis of the laser phase noise. To keep the power variations from producing thermal noise in excess of this, the power dissipation of the payload electronics will have to be controlled to $10 \mathrm{~mW} / \sqrt{\mathrm{Hz}}$ and the power dissipation of the photodiodes on the optical bench will have to be controlled to better than $50 \mu \mathrm{W} / \sqrt{\mathrm{Hz}}$. The needed control can be achieved with small heaters and voltage and current sensors. The spacecraft electronics do not need to be controlled to better than the $0.1 \%$ typical of flight-qualified units.

The secondary mirror of the telescope is supported from the primary by a graphite-epoxy spider with length $40 \mathrm{~cm}$ and thermal coefficient of expansion $0.4 \times 10^{-6} / \mathrm{K}$. The thermally-induced path-length variations using the thermal model were found to be less than $2 \mathrm{pm} / \sqrt{\mathrm{Hz}}$ at $1 \mathrm{mHz}$, and so are not a major source of noise.

The accelerations caused by changes in the mass distribution of the payload were assessed. The primary payload masses are the optical bench, the telescope, the payload electronics, and the laser/radiator combination. The proof mass acceleration noise caused by solar fluctuations was found to be less than $1 \times 10^{-16} \mathrm{~m} / \mathrm{s}^{-2} / \sqrt{\mathrm{Hz}}$ at $1 \mathrm{mHz}$. The acceleration noise due to thermal variations in the dimensions and component positions of the spacecraft body has not yet been assessed. 


\section{LASERS}

Lasers have extremely narrow beams that can survive long journeys through space. In addition, they are very stable in frequency (and phase) which is crucial to interferometry since phase "noise" appears just like gravitational waves. Furthermore, the infrared light has a frequency of $3 \times 10^{1.4} \mathrm{~Hz}$ which renders it immune from refraction caused by the charged particles (plasma) which permeate interplanetary space.

The lasers for LISA must deliver sufficient power at high efficiency, as well as being compact, stable (in frequency and amplitude), and reliable. The plan is to use solid-state diode-pumped monolithic miniature $\mathrm{Nd}$ :YAG ring lasers which generate a continuous $1 \mathrm{~W}$ infra-red beam with a wavelength of $1.064 / / \mathrm{m}$.

\section{Laser Frequency Noise}

The presence of laser frequency noise can lead to an error in the measurement of each arm length. If the arms are equal these errors cancel out but if they are unequal, the comparison of lengths used to search for gravitational waves may be dominated by frequency noise.

For an arm of length $L$ the phase difference between the outgoing and returning light of frequency ". is given by:

$$
\phi=\frac{4 \pi \nu L}{c} .
$$

Thus, for slow changes in $L$ and $\nu$,

$$
\delta \phi=\left[\frac{4 \pi}{c}\right](L \delta \nu+\nu \delta L)=\frac{4 \pi \nu L}{c}\left(\frac{\delta \nu}{\nu}+\frac{\delta L}{L}\right)
$$

where $\delta \omega$ is a phase fluctuation resulting from either a change $\delta L$ in arm length or a change $\delta \nu$ in frequency of the lascr. In fact a fractional change in frequency of $\delta \nu / \nu$ gives a signal equivalent to a fractional change in length of $\delta L / L$. Thus if the difference in two arm lengths is $\Delta x$ and the relative frequency stability of the laser is $\widetilde{\delta} \nu / \nu$ the smallest relative displacement which can be measured is given by:

$$
\widetilde{\delta x}=\Delta x \frac{\widetilde{\delta \nu}}{\nu}
$$

For the $5 \times 10^{6} \mathrm{~km}$ arms of LISA, a maximum value of $\Delta x$ of the order of $10^{5} \mathrm{~km}$ is likely. For a relative arm length measurement of $2 \times 10^{-12} \mathrm{~m} / \sqrt{\mathrm{Hz}}$, which is needed to achieve the desired overall sensitivity, a laser stability of $6 \times 10^{-6} \mathrm{~Hz} / \sqrt{\mathrm{Hz}}$ is required. In order to reach the desired sensitivity high precision frequency stabilisation has to be provided. The primary method of stabilisation is to lock the frequency of one laser in the system on to a Fabry-Perot cavity mounted on one of the craft and then to effectively transfer this stability to other lasers in the system by phase locking techniques. With the temperature fluctuations inside each craft limited in the region of $10^{-3} \mathrm{~Hz}$ to approximately $10^{-6} \mathrm{~K} / \sqrt{\mathrm{Hz}}$ by three stages of thermal insulation, a cavity formed of material of low expansion coefficient such as ULE allows a stability level of approximately $30 \mathrm{~Hz} / \sqrt{\mathrm{Hz}}$. This level of laser frequency noise is clearly much worse than the required $6 \times 10^{-6} \mathrm{~Hz} / \sqrt{\mathrm{Hz}}$ and a further correction scheme is required. Such a correction is provided by comparing the mean phase of the light returning in two adjacent arms with the phase of the transmitted light. The phase difference, measured over the time of flight in the two arms, allows an estimate of laser frequency noise to be made. For each arm $\widetilde{\delta \phi}=(4 \pi / c) L \widetilde{\delta \nu}$ (since $\nu \widetilde{\delta L} \ll L \widetilde{\delta \nu})$ and thus if the spectral density $\widetilde{\delta \phi}$ is measured, the spectral density $\widetilde{\delta \nu}$ can be estimated. 
The tolerable limit to laser intensity noise is to a lage extentset by the radiation pressure effects of the healm to the adjacent craft at a vertex, this beam being reflected off the proof mass in the accelerometer. We want few spurious accelerations of the proof mass above a level of $10^{-16} \mathrm{~m} \mathrm{~s}^{-2} / \sqrt{\mathrm{Hz}}$. For a proof mass of $1.3 \mathrm{~kg}$ and a reflected light power of $10 \mathrm{~mW}$, the proof mass will undergo a steady acceleration of $6 \times 10^{-11} 111 \mathrm{~s}^{-2}$. To keep the fluctuating acceleration $<10^{-16} \mathrm{~m} \mathrm{~s}^{-2} / \sqrt{\mathrm{Hz}}$ the power stability of the reflected light, and hence of the laser, must be better than $\widetilde{\delta P} / P=2 \times 10^{-6} / \sqrt{\mathrm{Hz}}$.

\section{On-Board Frequency Reference}

A further technical problem, that of the Doppler shift, occurs if there is a relative velocity between the two spacecraft in an arm, producing a frequency shift in the returned light and causing a beat signal when the phase comparison is made. For the particular orbits chosen, the relative velocities of the spacecraft in arm 3 will be up to about $15 \mathrm{~m} / \mathrm{s}$. The corresponding beat frequencies are then less than $15 \mathrm{MHz}$.

A signal from an ultra-stable oscillator (USO) is required in each arm to allow the Doppler beat fiequency to be reduced to a manageable level for later signal processing. The clock signal should be stable enough to contribute a level of phase noise less than that from an arm length change of $2 \times 10^{-12} \mathrm{~m} / \sqrt{\mathrm{H} \%}$. i.e. $1.2 \times 10^{-5} \mathrm{rad} / \sqrt{\mathrm{Hz}}$. The noise $\delta F$ of the clock frequency $F$ is related to the phase noise $\delta \phi$ at any frequency $f$ by $\delta F=f \times \delta \phi$, so at $10^{-3} \mathrm{~Hz}$ we require a clock with a noise $\overline{\delta F} \leq 1.2 \times 10^{-8} \mathrm{~Hz} / \sqrt{\mathrm{Hz}}$.

If the clock frequency is, say, $15 \mathrm{MHz}$, the required relative stability of the clock is approximately $8 \times 10^{-16} / \sqrt{\mathrm{Hz}}$, an Allan variance ${ }^{1}$ of $3 \times 10^{-17}$ at $10^{-3} \mathrm{~Hz}$. This demand is considerably stronger than can be fulfilled by any flight qualified USO currently available; for example, the one used on Mars Observer had an Allan variance of $2 \times 10^{-13}$ at $10^{-3} \mathrm{~Hz}$. The stability of the USO can, however, be improved to the desired level by modulating the clock frequency onto the laser light and stabilising this frequency to the arm length in a scheme analogous to that used to stabilise the laser frequency.

\section{DRAG-FREE AND ATTITUDE CONTROL}

An essential task of the spacecraft is to protect the mirrors from any disturbances which could jostle them around and create phase-signals that appear as gravitational waves. For example, consider the momentum of the light from the Sun which amounts to an average pressure of about $5 \times 10^{-6} \mathrm{~N} / \mathrm{m}^{2}$. The internal dynamics of the Sun lead to small variations - less than one percent - in this photon pressure, which occur at the low frequencies within LISA's range of interest. Although this variable photon pressure may seem rather small, if it were allowed to act on the cubical mirrors, the resulting motion would be $10^{4}$ times larger than the tiny motions due to gravitational waves that LISA is looking for.

By simply "wrapping a spacecraft around each one", the cubes are isolated from the solar pressure - but this is not the complete picture. When the solar pressure blows on the surface of the spacecraft, it will move relative to the freely-floating cube. Left alone, this motion would build up to unacceptable levels - in the extreme case, the cube would eventually "hit the wall". To stop this from happening, the relative motion can be measured very precisely by monitoring the change in electrical capacitance between the cube and electrodes mounted on the spacecraft. This measurement is then converted into a force-command which instructs thrusters mounted on the outer structure of the spacecraft, to fire against the solar pressure and keep

\footnotetext{
${ }^{1}$ For a clock with white frequency noise, the relationship between the Allan Variance and the relative frequency stability of the clock at a Fourier frequency $f$ is given by $\sigma_{\text {Alan }}=\sqrt{2 \ln 2} \times(\widetilde{\delta F} / F) \times \sqrt{f}$.
} 
the spacecraft centred on the cube.

This concept is, for historical reasons, known as "drag-free control", since it was originally invented in the 1960's to shield Earth-orbiting satellites from the aerodynamic drag due to the residual atmospheric gases. The method was first demonstrated on the TRIAD spacecraft, flown by the US Navy in 1972, where the dralgfiee controller designed at Stanford University in collaboration with the Johns Hopkins Applied Physics Laboratory, was effective in reducing the effects of atmospheric drag by a factor of $10^{3}$. Since then. the technique has undergone continued development, most notably for use on NASA's Gravity Probe B mission, which is the proposed space experiment to search for the relativistic precessions of gyroscopes orbiting the Earth.

The thrusters used on conventional spacecraft are far too powerful for LISA. The drag-free system only needs to develop a force of a few micro-newtons. Furthermore, the delivered force must be smoothly controllable so that the varying disturbance forces can be matched without introducing a further disturbance from the thrust system itself. Surprisingly, it is not a trivial task to build a thruster which generates such a small force and yet operates smoothly and does not consume too much power. By good fortune, ESA has been developing them for years, as an alternative to hydrazine rockets for station-keeping of communication satellites.

They are called FEEPs, for Field Emission Electric Propulsion. They operate by accelerating ions in an electric field, and ejecting them to develop the thrust.

\section{ULTRASTABLE STRUCTURES}

The small variations in the intensity of sunlight will cause fluctuations in the heat-load applied to the spacecraft. This could lead to thermal gradients across the optical bench, which would upset the stability of the laser cavity. To obtain the required thermal stability, most structural elements are made from carbon-epoxy which has a thermal expansion coefficient of $4 \times 10^{-7} / \mathrm{K}$ and the optical bench is made from ULE, which has a temperature coefficient at least a factor 4 lower over the possible temperature range of the LISA payload. Furthermore, low emissivity coatings are used on most surfaces inside the spacecraft and a thermal shield surrounds the payload cylinder, in order to provide isolation from the temperature variations of the spacecraft skin that is exposed to the Sun. These shields are only effective against heat fluctuations faster than a few hours to half a day. The slower variations will get through, thus making the sensitivity of LISA deteriorate rapidly below roughly $10^{-4} \mathrm{~Hz}$. The use of carbon-epoxy structures also minimises any thermally-induced mechanical distortions which could produce physical changes in the optical path-length, as well as local gravitational disturbances on the mirror-cubes.

\section{DATA TRANSMISSION}

Each spacecraft will be equipped with two (one spare) $\mathrm{X}$-band transponders with steerable $30 \mathrm{~cm}$ high-gain antennas for communication with the Earth. On average, the transmissions will require about eight hours per day, at a data rate of roughly 600 bits per second. The entire LISA data set, after a nominal two-year mission, will be stored on about a hundred CD-ROMs. 